\title{
Automated Diagnosis of Knee Pathology Using Sensory Data
}

\author{
Majid Janidarmian, Katarzyna Radecka and Zeljko Zilic \\ Department of Electrical and Computer Engineering, McGill University, Montreal, Canada \\ majid.janidarmian@mail.mcgill.ca, \{katarzyna.radecka, zeljko.zilic\}@ mcgill.ca
}

\begin{abstract}
In order to early diagnosis and treatment of knee abnormalities, in this study an automated diagnosis system using wearable EMG and goniometer sensors is proposed. Eight different classification techniques are investigated with a set of time-domain features. The experiments are conducted with 22 subjects' data and the best accuracy of $97.17 \%$ is achieved based on the Bagged Decision Trees classifier. We have also evaluated the classifications quality with Fixed-size Overlapping Sliding Window (FOSW) segmentation technique where SVM and Bagged Decision Trees classifiers could obtain the accuracy of $100 \%$ in distinguishing healthy subjects from people with knee abnormality.
\end{abstract}

Keywords-knee pathology; classification; feature extraction; surface EMG; goniometer

\section{INTRODUCTION}

Since the knee joint bears the heaviest weight of the human body, it is the most susceptible body part to damage [1]. Knee related injuries in the US continue to be the most common career ending injuries in sports [2]. According to National Center for Health Statistics report [3], approximately 19 million visits were made to physicians because of knee abnormalities in 2003. There are different methods for knee disorders diagnosis; however during early stages of deterioration, most patients typically have no symptoms of changes on standard diagnostic tests such as x-rays [4]. With the growth of sensor technology and the analysis methods, diagnosis systems based on wearable sensors carry the advantages of simple setup, high reliability and accuracy as well as providing useful information for health-related applications. One area of interest is applying EMG sensors which record the electrical activity, provided by skeletal muscles. When muscles are active, the sensors produce an electrical current which is usually relative to the level of muscle activity [2]. EMG was introduced during the first half of the 20th century, and the premier major studies on EMG during walking were performed in the 1940s and 1950s [5]. [6] shows that surface EMG signals provide reliable data for clinical evaluation of muscle activity since they provide valuable information about muscle contraction and fatigue.

Designing a safe and non-invasive system using wearable sensors for knee pathology can play an important role in both diagnosis and treatment. Several classification schemes have been used for medical diagnosis through analysis of data collected by different on-body sensors. In this paper, we are going to use various classifiers to accurately identify people who suffer from a knee abnormality. First, data collecting from EMG electrodes and goniometer are divided into windows of size $n$, and features are derived from each separate window of data. Then, various classifiers are used to distinguish healthy subjects from people with knee abnormality. This system could be potentially used in the patient's home or in a physical therapy office. In fact, early identification through such system and timely treatment of exacerbations can decrease the hospital admissions and slow deterioration while reducing disease costs [7].

\section{SENSORS DATA ANALYSIS}

\section{A. Data Collection and Setup}

In this paper, we have used EMG (electromyography) and goniometer sensors data which recently released in a wellknown machine learning repository [8]. Twenty two individuals were recruited in the study. Half of the subjects had different knee abnormalities which previously diagnosed by a professional. Each subject were instructed to perform three activities i.e. gait, knee extension and flexion in the seated and standing positions. Surface EMG electrodes were measured at $1 \mathrm{KHz}$ and placed on four muscles crossing the knee: vastus medialis (VASMED), semitendinosus (STEN), biceps femoris (BIFEM), rectus femoris (RECFEM) as shown in Fig. 1.

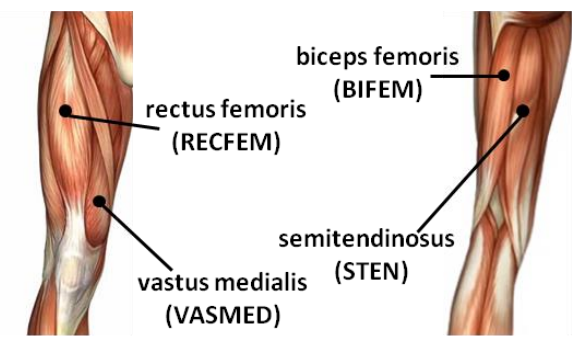

Fig. 1. Four muscles crossing the knee where surface EMG electrodes are placed

The electrodes connect to DataLOG MWX8 [9] which can be worn on the arm or leg. It is capable of displaying and analyzing in real time within PC using Bluetooth and also store data into the Micro SD card. It is worth noting that the raw EMG data is used and no filtering has been applied to reduce artifacts. In addition to the electrodes, a Biometrics' goniometer [9] was utilized to accurately measure the joint movement of the knee and its data was extrapolated to 1000 samples per second. The goniometer is operated by measuring 
the change in the physical signal resulting from the angular changes. For further details of the study, the reader is directed to [8].

\section{B. Feature Extraction}

Feature extraction is to obtain the important characteristics of a data and represent them into a set of features [10]. However, stream of sensory data requires segmentation in order to facilitate effective feature extraction. The first popular method is dividing the data stream into windows of fixed length with no inter-window gaps and no degree of overlap between adjacent windows (Fixed-size Non-overlapping Sliding Window (FNSW)) [11]. The second method is Fixedsize Overlapping Sliding Window (FOSW) which is similar to FNSW except that the windows overlap during segmentation [12]. Due to their low complex implementations and reasonable performance, we have used these techniques.

The features are extracted from each separate window of data and then used as inputs to the classifiers. In Table I, we describe the most representative techniques in time-domain for extracting signal features. Time-domain features are derived directly from a window of sensor data and are typically statistical measures.

\section{Classification Algorithms}

The raw sensors data are binary labeled based on the subjects who can be healthy or have knee pathology. We have employed a wide range of classification algorithms including: Decision Tree (DT), Bagged Decision Trees (TB), Generalized Linear Model (GLM), Discriminant Analysis (DA), k-Nearest Neighbors (kNN), Support Vector Machines (SVM), Naïve Bayes (NB) and Artificial Neural Networks $(\mathrm{NN})$.

The decision tree which is similar to hierarchical classifications has been applied to a wide range of problems. It is a very successful technique for supervised classification learning [13]. TB builds an ensemble of classification trees (50 trees in this paper) and uses bagging to combine the predictions [14]. $\mathrm{kNN}$ algorithm is used for classification problems based on the $\mathrm{k}$ closest training examples in the feature space $(\mathrm{k}=10)$. NB is a simple probabilistic classifier according to the Bayes' theorem. A more general version of the naïve Bayesian is discriminant analysis, where crosscorrelations between features are taken into account. SVM is based on finding optimal separating decision hyperplanes between classes with the maximum margin between patterns of each class [15]. In our experiments, we have used the polynomial kernel function of order 3 for SVM classifier.

TABLE I. TIME SERIES FEATURES

\begin{tabular}{|c|c|c|c|}
\hline Feature & Description & Feature & Description \\
\hline Mean & $\mu_{s}=\frac{1}{\mathrm{n}} \sum_{\mathrm{i}=1}^{\mathrm{n}} \mathrm{s}_{\mathrm{i}}$ & Zero crossings & $\begin{array}{l}\text { Number of times the signal crosses its } \\
\text { zero/median }\end{array}$ \\
\hline Minimum & $\underset{i=1: n}{\min }(\mathrm{S})=\min \mathrm{s}_{\mathrm{i}}$ & Skewness & $\frac{1}{n \sigma_{s}^{3}} \sum_{i=1}^{n}\left(s_{i}-\mu_{s}\right)^{3}$ \\
\hline Maximum & $\max \underset{i=1: n}{(S)}=\max _{i}$ & Kurtosis & $\frac{1}{n \sigma_{s}^{4}} \sum_{i=1}^{n}\left(s_{i}-\mu_{s}\right)^{4}$ \\
\hline Median & $\begin{array}{l}\operatorname{median}_{i=1: n} s_{i} \\
\text { is the median value of the elements in } S\end{array}$ & Signal power & $\sum_{i=1}^{n} s_{i}^{2}$ \\
\hline Standard Deviation & $\sigma_{s}=\sqrt{\frac{1}{\mathrm{n}} \sum_{\mathrm{i}=1}^{\mathrm{n}}\left(\mathrm{s}_{\mathrm{i}}-\mu_{s}\right)^{2}}$ & Peak intensity & $\begin{array}{l}\text { The number of signal peaks within a } \\
\text { certain period of time }\end{array}$ \\
\hline $\begin{array}{l}\text { Coefficients of } \\
\text { variation }\end{array}$ & $c_{v}=\frac{\sigma_{s}}{\mu_{s}}$ & $\begin{array}{l}\text { Lag-one- } \\
\text { autocorrelation }\end{array}$ & $\frac{\sum_{i=1}^{n-1}\left(s_{i}-\mu_{s}\right)\left(s_{i+1}-\mu_{s}\right)}{\sum_{i=1}^{n}\left(s_{i}-\mu_{s}\right)^{2}}$ \\
\hline $\begin{array}{l}\text { Peak-to-peak } \\
\text { amplitude }\end{array}$ & $\max (S)-\min (S)$ & $\begin{array}{l}\text { Inter-axis Correlation } \\
\text { Coefficient }\end{array}$ & $\frac{\sum_{i=1}^{n}\left(s_{i}-\mu_{s}\right)\left(p_{i}-\mu_{p}\right)}{\sqrt{\sum_{i=1}^{n}\left(s_{i}-\mu_{s}\right)^{2} \sum_{i=1}^{n}\left(p_{i}-\mu_{p}\right)^{2}}}$ \\
\hline Percentiles & Percentile $(S, p), p=10,25,50,75,90$ & $\begin{array}{c}\text { Root Mean Square } \\
\text { (RMS) }\end{array}$ & $\sqrt{\frac{\sum_{i=1}^{n} s_{i}^{2}}{n}}$ \\
\hline Interquartile range & Percentile $(S, 75)-$ Percentile $(S, 25)$ & $\begin{array}{c}\text { Trapezoidal } \\
\text { numerical integration }\end{array}$ & $\begin{array}{c}\int_{1}^{n} S(x) d x \text { using Multiple Segment } \\
\text { Trapezoidal Rule }\end{array}$ \\
\hline
\end{tabular}




\section{EXPERIMETNAL RESULTS AND DISCUSSIONS}

\section{A. Data Segmentation Analysis}

All methods were trained with data collected by the electrodes and goniometer sensors. First, we used FNSW as the segmentation technique and Fig. 2 shows the classifications accuracies for a range of window sizes with 10-fold crossvalidation. The best accuracies of $92.14 \%, 97.17 \%, 87.95 \%$, $88.73 \%, 92.38 \%, 80.08 \%, 96.14 \%$ and $88.06 \%$ were achieved by DT, TB, GLM, DA, kNN, NB, SVM and NN classifiers, respectively. We also investigated another common segmentation technique called FOSW with five different overlap percentages. The classifiers accuracies with respect to the window size and the percentage of adjacent windows overlap for feature extraction are shown in Fig. 3. As can be seen, this technique significantly improves the accuracy of all classifiers; and two classifiers TB and SVM yield the highest classification accuracy $100 \%$ with windows size $5.5 \mathrm{sec}$ and overlap size $90 \%$. The red points in Fig. 3 indicate the best obtained accuracies for different methods and windows parameters. These results also summarized in Table II.

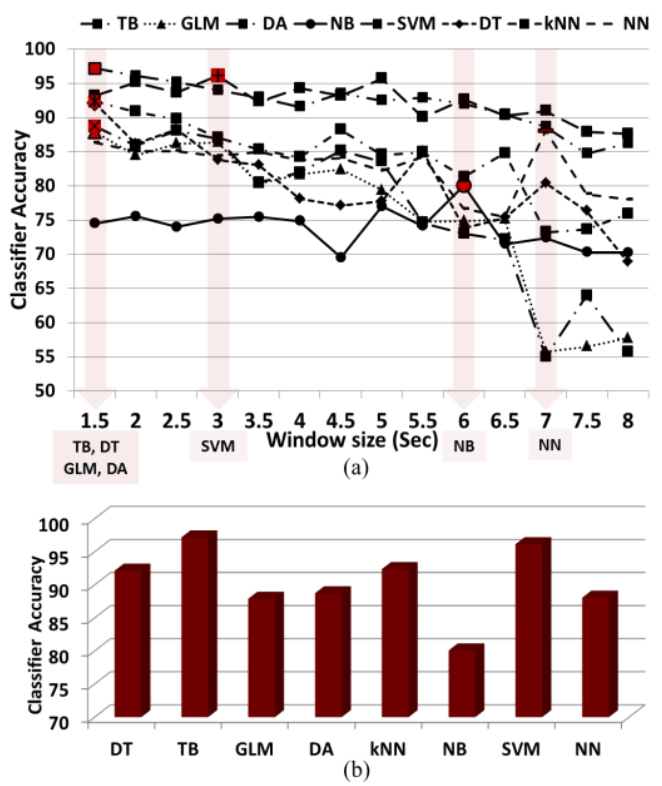

Fig. 2 Classification accuracy of 8 methods with different window sizes using the FNSW segmentation technique

\section{B. Number of Sensory Nodes}

As mentioned earlier, data collected form four surface EMG and one goniometer sensors are used for features extraction and classification. However, it is important to minimize sensors redundancy as this can result in reducing computational and cost demand. Therefore, in this section, we empirically investigated the classifications accuracies when the number of the sensors is reduced. Table III reports effective results showing that we can keep the accuracy $100 \%$ with employing only one sensor either the EMG at vastus medials muscle or the goniometer. It is worth mentioning that the overlap percentage of $90 \%$ is kept for this analysis. Moreover, as observed from the results, accuracy of some classifiers can be increased by utilizing less sensors.
TABLE II. THE HIGHEST ACCURACIES FOR WINDOW OVERLAP AND WINDOW SIZE COMBINATIONS IN DIFFERENT CLASSIFIERS

\begin{tabular}{cccc}
\hline Method & $\begin{array}{c}\text { Window } \\
\text { Size(Sec) }\end{array}$ & $\begin{array}{c}\text { Window } \\
\text { Overlap } \\
\text { Value (\%) }\end{array}$ & $\begin{array}{c}\text { Highest } \\
\text { Classification } \\
\text { Accuracy (\%) }\end{array}$ \\
\hline DT & 4 & 90 & 99.75 \\
TB & $\mathbf{5 . 5}$ & $\mathbf{9 0}$ & $\mathbf{1 0 0}$ \\
DA & 3 & 75 & 99.25 \\
GLM & 3 & 75 & 99.43 \\
kNN & 7 & 90 & 97.72 \\
NB & 4.5 & 90 & 96.44 \\
SVM & $\mathbf{5 . 5}$ & $\mathbf{9 0}$ & $\mathbf{1 0 0}$ \\
NN & 6 & 90 & 99.72 \\
\hline
\end{tabular}

TABLE III. LIST OF CLASSIFIERS WHICH KEEP THE ACCURACY OF 100\%

\begin{tabular}{|c|c|c|c|c|c|c|}
\hline S1 & S2 & S3 & S4 & S5 & Method & $\begin{array}{c}\text { Window size } \\
\text { (Sec) }\end{array}$ \\
\hline & & $\mathbf{X}$ & & & TB & $\mathbf{5 . 5}$ \\
\hline $\mathrm{X}$ & & $\mathrm{X}$ & & & $\mathrm{TB}$ & 4 \\
\hline $\mathrm{X}$ & & $\mathrm{X}$ & & & $\mathrm{TB}$ & 5.5 \\
\hline $\mathrm{X}$ & & $\mathrm{X}$ & & & $\mathrm{TB}$ & 6 \\
\hline & $\mathrm{X}$ & $\mathrm{X}$ & & & $\mathrm{TB}$ & 5.5 \\
\hline & $\mathrm{X}$ & $\mathrm{X}$ & & & $\mathrm{NN}$ & 6 \\
\hline & $\mathrm{X}$ & $\mathrm{X}$ & & & $\mathrm{NN}$ & 6.5 \\
\hline & & $\mathrm{X}$ & $\mathrm{X}$ & & $\mathrm{TB}$ & 5.5 \\
\hline & & & & $\mathbf{X}$ & TB & $\mathbf{6 . 5}$ \\
\hline & & $\mathrm{X}$ & & $\mathrm{X}$ & $\mathrm{DT}$ & 5.5 \\
\hline & & $\mathrm{X}$ & & $\mathrm{X}$ & $\mathrm{TB}$ & 5.5 \\
\hline & & $\mathrm{X}$ & & $\mathrm{X}$ & $\mathrm{TB}$ & 6 \\
\hline & & $\mathrm{X}$ & & $\mathrm{X}$ & $\mathrm{GLM}$ & 6.5 \\
\hline & & $\mathrm{X}$ & & $\mathrm{X}$ & $\mathrm{DA}$ & 6.5 \\
\hline
\end{tabular}

$\mathrm{S} 1$ is the electrode located on rectus femoris, S2 is the electrode located on biceps femoris, S3 is the electrode located on vastus medials and S4 is the electrode located on semitendinosus. S5 is the goniometer

\section{FEATURE SELECTION}

The problem of finding an optimal subset of features out of all extracted features that best discriminate between classes is called feature selection [16]. It not only reduces the dimension, but also avoids misleading the classifier with redundant or irrelevant features. There are many feature selection algorithms in the literature [16]; however, we found each feature importance through permuting the feature across the out-of-bag observations and measuring the increase in prediction error. Then, the assessment of top five important features led us to choose only a set of 2 features i.e. maximum and interquartile range while classification accuracy remains $100 \%$.

\section{CONCLUSION}

In this paper, eight classification algorithms were analyzed for distinguishing healthy subjects from people with knee abnormality. These techniques used a large set of real data from 22 subjects. The surface EMG sensors were mounted on four muscles of knee. Besides a goniometer was considered to intervene the impacts of joint angles in the diagnosis methodology. The accuracy of $100 \%$ was achieved in this case study while using either the EMG on vastus medials muscle or the goniometer. We also investigated the effect of changing the number of sensors, segmentation technique, window size and overlap percentage. 


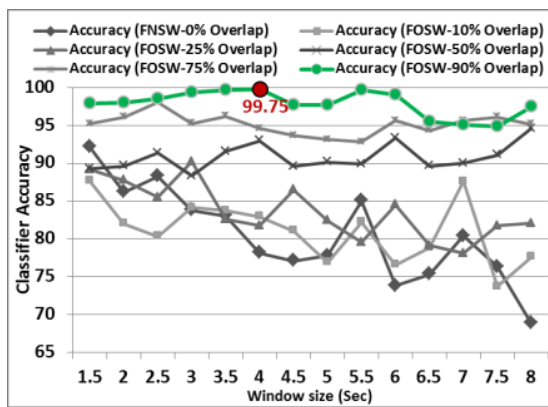

(a)

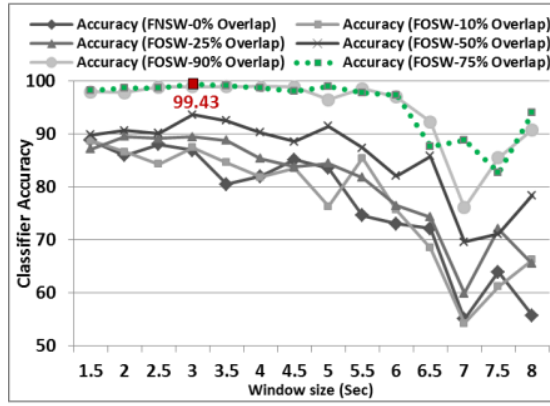

(d)

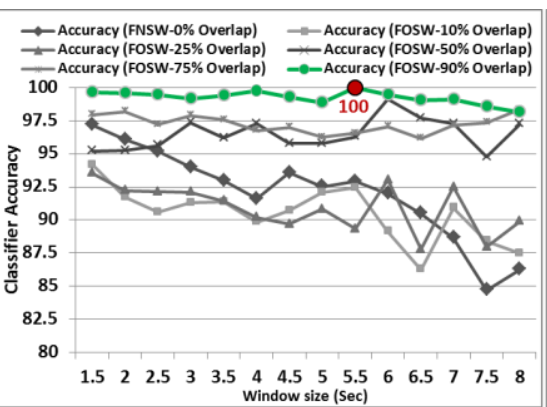

(b)

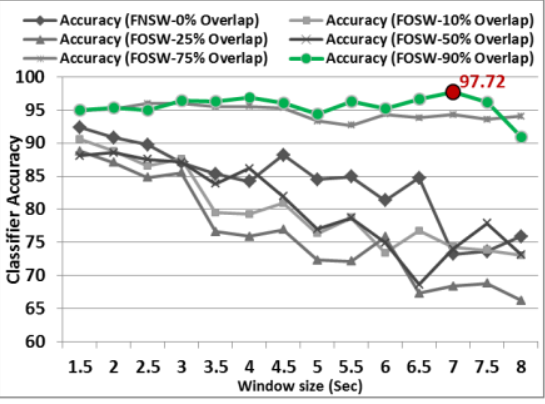

(e)

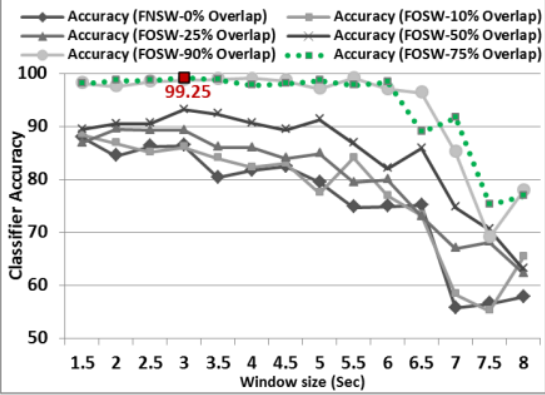

(c)

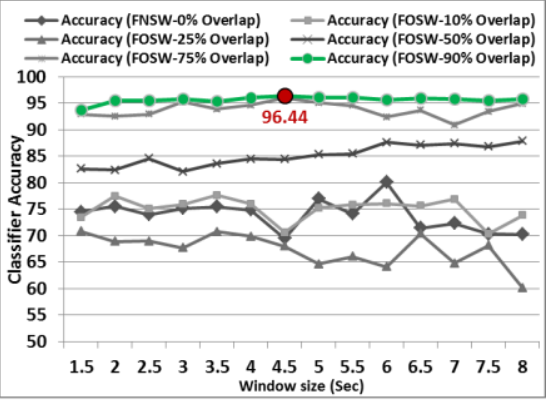

(f)

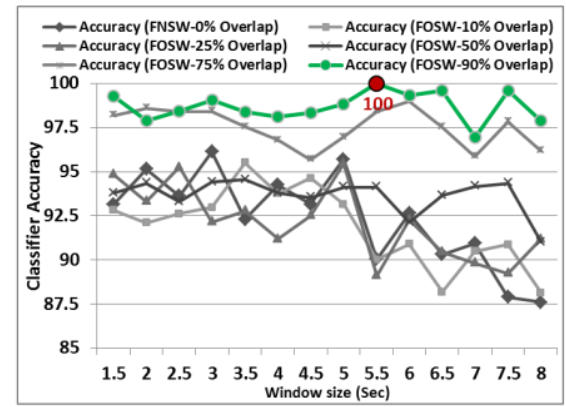

(g)

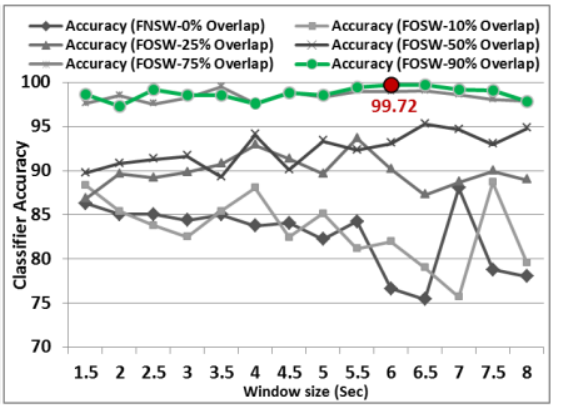

(h)

Fig. 3. The accuracies for window overlap and window size combinations in (a)DT, (b)BT, (c)DA, (d)GLM, (e)kNN, (f) NB, (g) SVM, (h)NN classifiers

\section{REFERENCES}

[1] C. Po-Chao, H .Chih-Ning, C. I-Chun, C. Chia-Tai, "A Rehabilitation Exercise Assessment System Based on Wearable Sensors for Knee Osteoarthritis," International Conference on Smart Homes and Health Telematics, ICOST 2013, pp 267-272, June 2013.

[2] Caitlin Clancy, Kartik Kesavabhotla, Chris O’Conor, Eileen Sun, Qian Wei, Clark Hung, "Orthometrics," Design Summary.

[3] National Center for Health Statistics, National Ambulatory Medical Care Survey, "Using all three possible reason-for-visit codes listed," the AAOS Department of Research and Scientific Affairs, 2003.

[4] Frank CB, Rangayyan RM, Bell GD, "Analysis of knee joint sound signals for non-invasive diagnosis of cartilage pathology," IEEE Engineering in Medicine and Biology Magazine, pp 65-68, 1990.

[5] A. Steindler, "A historical review of the studies and investigations made in relation to human gait," J. Bone Joint Surg. 1953, 35A, 540-542.

[6] JR. Cram, "Clinical EMG: Muscle Scanning for Surface Recordings," Biofeedback Institute of Seattle, pp 81-83, 1986.

[7] T. Wilkinson, G. Donaldson, J. Hurst, T. Seemungal, J. Wedzicha, "Early therapy improves outcomes of exacerbations of chronic obstructive pulmonary disease," American J. Respir. Crit. Care Med. 2004, 169, 1298-1303.

[8] O. F. A. Sanchez, J. L. R. Sotelo, M. H. Gonzales, G. A. M. Hernandez, "EMG dataset in Lower Limb Data Set", UCI machine learning repository, avaiable: 2014-02-05.

[9] http://www.biometricsltd.com
[10] N. C. Krishnan, C. Juillard, D. Colbry, and S. Panchanathan, "Recognition of hand movements using wearable accelerometers," JAISE, vol. 1, no. 2, pp. 143-155, 2009.

[11] S.J. Preece, J.Y. Goulermas, L.P.J. Kenney, D. Howard, K. Meijer, and R. Crompton, "Activity identification using body-mounted sensors-a review of classification techniques," Physiol. Meas. 30(4) (2009) vR1R33

[12] Keogh, E.; Chu, S.; Hart, D.; Pazzani, M. "An Online Algorithm for Segmenting Time Series," In Proceedings of the International Conference on Data Mining, San Jose, CA, USA, 29 November-2 December 2001; pp. 289-296.

[13] Avci, Akin; Bosch, Stephan; Marin-Perianu, Mihai; Marin-Perianu, Raluca; Havinga, Paul, "Activity Recognition Using Inertial Sensing for Healthcare, Wellbeing and Sports Applications: A Survey," 23rd International Conference on Architecture of Computing Systems (ARCS), pp.1,10, 2010.

[14] Pardos, Z.A., Heffernan, N. T., "Using HMMs and bagged decision trees to leverage rich features of user and skill from an intelligent tutoring system dataset,". In Proceedings of the KDD cup workshop, pp. 24-35, 2010.

[15] A. Roshan Fekr, M. Janidarmian, K. Radecka, Z. Zilic, "A medical cloud-based platform for respiration rate measurement and hierarchical classification of breath disorders," Sensors 2014, 14, 11204-11224.

[16] Khusainov, R.; Azzi, D.; Achumba, I.E.; Bersch, S.D."Real-Time Human Ambulation, Activity, and Physiological Monitoring: Taxonomy of Issues, Techniques, Applications, Challenges and Limitations," Sensors 2013, 13, 12852-12902. 